\section{C SAFETY AT WORK: AN ISSUE OF DAILY MANAGEMENT BEHAVIOR}

Mark Fuellemann. Practice \& Experience Ltd., Baden, Switzerland

\subsection{6/oemed-2018-ICOHabstracts. 1128}

Do we know any employee who wants to have an accident and hurt himself while driving to work in the morning? The answer obviously is 'No'. Nevertheless, our traffic radios report accidents on the road every single morning, accidents that took place although nearly all drivers know how to drive safely. It is equally obvious that nobody wants to suffer from an accident at work, the difference being that on the road we are fully responsible for our safety whereas at work there is the additional responsibility of management which results from the basic business principle of 'pacta sunt servanda'. For his performance, we promise the worker among other things to keep him safe. The question now becomes 'what can management do?'. Some fifty years ago we stopped blaming the employee for violating rules (at times it is still done!) and we turned to behavior based safety, meaning that management has to influence the workers so that they will work - meaning behave - in a safe way. As a consequence, we have to figure out why people could and would behave in an unsafe way. The main reason for this is that safety is not - as sometimes written in corporate documents - our most important objective. We do not start work to be safe but to accomplish things. We seek that satisfaction of something well done - and when I write 'we' I mean everybody. Most worker I have met during the last 25 years want to accomplish something, for different reasons. Safety however is not an accomplishment. As long as no incidents happen, safety is neither perceived nor measured. We perceive changes; safety staying safety is not on our radar screen and therefore is being pushed in the background of our mind. Additionally, most of us believe that incidents happen only to others: young people think that incidents take place when older people are slow to react and older people tend to think that younger people are rash and do not evaluate risks properly. Consequently, we have a case of change management before us. Using a model developed by the INSEAD professor Jean-François Manzoni I asked a number of management teams which was the most important lever to influence the behavior of workers. The outcome was very clear: it is the behavior of management. But the same management teams indicated that they spent very few hours influencing safety for a variety of reasons, not the least one being not knowing how to do it. Therefore, we first have to change the behavior of managers regarding their role in safety at work. Managers must recognize that they should be the teachers, the coaches, the motivators and the mentors of their people regarding safety at work. And this combination has to be applied daily, the most important activity being delivering the daily Safety Moment at the start of work and the very frequent Safety Observation Tour by all managers (all levels and all functions) to make safety at the worker level an integral part of work life.

\section{7d RECOGNITION OF OCCUPATIONAL DISEASES IN SWITZERLAND: WHY HAVE WE FALLEN BEHIND, AND HOW CAN WE IMPROVE THE SYSTEM TO BETTER PROTECT WORKERS?}

\footnotetext{
1,2Halshka Graczyk* ${ }^{2}$ Irina Guseva Canu. 'Department of Public Health, Government of the Canton of Vaud, Switzerland; 'Institute for Work and Health, University of Lausanne, Switzerland
}

Occupational disease lists are essential legal mechanisms for recognizing pathologies related to hazardous workplace exposures. In Switzerland, 1.1 million workers are affected by work-related health problems annually. In terms of financial repercussions, the situation is not negligible: the cost of occupational diseases amounts to at least CHF 20 billion per year. However, there is currently no centralized database on workers' exposures to occupational hazards in Switzerland; nor a national register of chronic effects due to occupational exposures. Moreover, Switzerland has made limited progress in its stategy for updating the recognized occupational disease list. In October 2017, a revision to the occupational disease list was proposed for the first time in ten years, highlighting the urgent need to ameliorate the system of occupational disease reporting and recognition in Switzerland. This review will focus on the importance of occupational disease lists towards occupational safety and health strategies and prevention efforts, while highlighting the unique case of Switzerland, with the aim to gather evidence and good practices towards the improvement of its own occupational disease list.

\section{Occupational Toxicology}

\section{PROFICIENCY TESTING FOR QUALITY ASSURANCE OF BIOMONITORING DATA}

Thomas Göen*, Barbara Schaller, Hans Drexler. Inst. of Occupational, Social and Environmental Medicine, University of Erlangen-Nuremberg, Erlangen, Germany

\subsection{6/oemed-2018-ICOHabstracts. 1130}

Introduction Biomonitoring is the most powerful approach for a credible risk assessment of chemical exposure at the workplace. Because adequate certified reference materials are generally not available, the participation in inter-laboratory comparison tests is the exclusive feasibility to receive information on comparability and accuracy of biomonitoring laboratories. The presentation displays the concept of the German External Quality Assessment Scheme (GEQUAS), the requirements and limitation for the adjustment of the programme as well as an extract of the results.

Methods Parameter scheme, participation quota, target values, tolerance ranges and rates of success (certificate number/participant number) were extracted from the data and results of the last 10 runs of the proficiency test programme. Each parameter was provided in two different concentrations. Generally, the target values and the tolerance ranges were estimated by the results of several so-called reference laboratories. A successful participation was certified for a parameter if the results of the participant were found to be inside the tolerance ranges of both concentrations.

Results 162 parameters were provided in the last run $(7$ metals in blood, 36 inorganic parameters in urine, 12 metals in plasma, 10 mercapturic acids in urine, 11 phenolic compounds in urine, 7 amines in urine, 12 solvents in urine (headspace technique), 36 other organic parameters in urine, 11 solvents in blood (headspace technique), 15 halogenated hydrocarbons in serum, $5 \mathrm{~N}$-terminal valine adducts in globin). In last runs about 200 laboratories participated, of which three-fourth were located outside Germany. The rate of success ranged for most parameters between 50\% and 100\% (mean 75\%). Poor rate of success occurred often when conjugates had to hydrolyse before analysis. 
Discussion GEQUAS enables proficiency testing for the most biomonitoring parameters which are used for the exposure assessment at workplaces (metals, solvents, pesticides, new emerging chemicals). Levels and specification are carefully adjusted to the practice.

\section{MIXIE, A TOOL TO IMPROVE ASSESSMENT OF CHEMICAL RISK IN CASE OF MULTIPLE EXPOSURE}

L Coates*, N Bertrand, S Binet, P Campo, F Clerc, B La Rocca, F Pillière. National Research and Safety Institute for the Prevention of Occupational Accidents and Diseases (INRS)

\subsection{6/oemed-2018-ICOHabstracts.1131}

Introduction Multiple exposure to chemicals is a common situation in workplaces. However, most methods used to evaluate chemical risk do not consider the potential effects of mixtures. The aim is to present a tool helping to evaluate chemical risk in case of multiple exposure.

Methods MiXie is a web tool (http://www.inrs-mixie.fr/http:// www.inrs-mixie.fr/) developed in Quebec in 1997 and adapted to the French context by the French National Research and Safety Institute for the prevention of occupational accidents and diseases (INRS). It helps industrial hygienists to assess the potential risk of multi-exposure. Additivity of effects is the basic assumption.

Results Whenever measurements of atmospheric concentrations are provided, MiXie calculates the exposure index of the mixture (i.e. the sum of the ratios between each concentration measured and its occupational exposure limit value $x$ 100). If this index exceeds $100 \%$, MiXie signals that there is a potential risk for certain organs, even though each limit value is respected.

When measurements of atmospheric concentrations are not provided, MiXie highlights the common effects classes of the substances present and warns about a potential risk of additive effects.

If the mixture contains a substance belonging to the 'cancer' or 'sensitizer' effect class, additivity does not apply and MiXie warns the industrial hygienist regardless of the concentration measured.

Conclusion The MiXie database helps to identify potential risk situations related to multi-exposure to chemicals. Such situations may go unnoticed with a monosubstance approach. But MiXie users should be aware of its limits: additivity does not apply to all situations, the number of substances is restricted (130), etc. Experts are working to improve the tool by increasing the number of substances, making it more userfriendly, etc.

\section{EFFECTS OF METAL-RICH PARTICULATE MATTER EXPOSURE ON EPSTEIN-BARR VIRUS AND HUMAN ENDOGENOUS RETROVIRUS W (HERV-W) METHYLATION HEALTHY STEEL-WORKERS}

\footnotetext{
1,2V Bollati ${ }^{*},{ }^{1,2} \mathrm{M}$ Bonzini, ${ }^{1} \mathrm{R}$ Mercorio, 'L Angelici, ${ }^{1} \mathrm{~J}$ Mariani, 'S Delbue, ${ }^{4} \mathrm{P}$ Apostoli,

1,2 AC Pesatori. 'EPIGET Lab - Dept. Clinical Sciences and Community Health, Università degli Studi di Milano, Milan, Italy; ${ }^{2}$ Fondazione IRCCS Ca' Granda Ospedale Maggiore Policlinico, Occupational Medicine Unit, Milan, Italy; ${ }^{3}$ Dept Biomedical, Surgical and Dental Sciences, Università degli Studi di Milano, Milan, Italy; ${ }^{4}$ Dept Experimental and Applied Medicine, University of Brescia, Brescia, Italy
}

10.1136/oemed-2018-ICOHabstracts.1132
Introduction Inhaled particulate pollutants have been shown to produce systemic changes in DNA methylation. Global hypomethylation has been associated to viral sequence reactivation, possibly linked to the activation of pro-inflammatory pathways occurring after exposure. We aimed at evaluating the effects of PM exposure on DNA methylation of the Wp promoter of the Epstein-Barr Virus (EBV-Wp) and the promoter of the human-endogenous-retrovirus w (HERV-w), chosen as a paradigm of an exogenous virus and an endogenous retroviral sequence, in workers in an electric furnace steel plant with well-characterised exposure to metal-rich particulate matter.

Methods We measured EBV-Wp and HERV-w DNA methylation through bisulfite PCR Pyrosequencing on peripheral blood leukocytes DNA obtained from 63 male healthy workers, on the first day of a work week (baseline, after 2 days off work) and after 3 days of work (post-exposure). We determined individual exposure to inhalable particles and metals for all subjects. Paired t-test was used to compare baseline and post-exposure samples. Linear mixed models were fitted to evaluate the association between metal-rich particle exposure and DNA methylation.

Results Comparing samples obtained at baseline and after 3 days of work, the mean methylation of EBV-Wp was significantly higher at baseline compared to post-exposure (baseline $=56.7$; postexposure $=47.9 ; \mathrm{p}$-value $=0.009$ ), whereas the mean methylation of HERV-w did not significantly differ. In a regression model adjusted for age, body mass index and smoking, nickel, arsenic and lead had a positive association with EBV-Wp methylation (nickel: $\beta=16.16, \quad$ p-value $<0.001$; arsenic: $\quad \beta=13.0, \quad p$-value $=0.02$; lead: $\beta=16.53$, $p$ value $<0.001)$.

Conclusions The difference observed comparing baseline and postexposure samples may be suggestive of a rapid change in EBV methylation induced by air particles, while correlation between EBV methylation and metal exposure may represent an adaptive mechanism that should be further characterised in future investigations.

\section{STEROID HORMONES, MELATONIN AND VITAMIN D IN FEMALE HOSPITAL NURSES WORKING WITH '1-1-1' RAPID CYCLE SHIFT}

C Maggioni, E Crespi, E Polledri, R Mercadante, G Costa, S Fustinoni ${ }^{*}$. Dipartimento di Scienze Cliniche e di Comunità, Università degli Studi di Milano e Fondazione IRCCS Ca' Granda Ospedale Maggiore Policlinico, Via S. Barnaba, 8- 20122 Milano, Italia

\subsection{6/oemed-2018-ICOHabstracts.1133}

Introduction Shiftwork that disrupts circadian rhythms has been classified as probable carcinogenic to humans by IARC (2007). Among possible mechanisms of this effect, the modification of hormone homeostasis has been advocated. Aim of the present study was to evaluate the influence of night-shift work on the levels of steroid hormones, melatonin, and serum vitamin $\mathrm{D}$ in hospital female nurses.

Methods Ninety-five female hospital nurses were recruited: 45 performing ' $1-1-1$ ' fast rotating shift schedule on 5 day cycle (morning - afternoon - night - rest - rest) and 50 working only on dayshift, as controls. Specimens were collected to measure 13 serum steroid hormones, salivary cortisol, cortisone and melatonin in morning and evening samples, and serum vitamin D. All markers were assayed by liquid chromatography coupled with triple quadrupole mass spectrometry. 